\title{
Dynamical Compactification of Extra Dimensions in the Euclidean IKKT Matrix Model via Spontaneous Symmetry Breaking
}

\section{Konstantinos N. Anagnostopoulos}

Physics Department, National Technical University of Athens, Zografou Campus, GR-15780 Zografou, Greece

E-mail: konstantemail.ntua.gr

\section{Takehiro Azuma}

Institute for Fundamental Sciences, Setsunan University, 17-8 Ikeda Nakamachi, Neyagawa, Osaka, 572-8508, Japan

E-mail: azumaempq.setsunan.ac.jp

\section{Yuta Ito}

National Institute of Technology, Tokuyama College, Gakuendai, Shunan, Yamaguchi 745-8585, Japan and KEK Theory Center, High Energy Accelerator Research Organization, 1-1 Oho,

Tsukuba, Ibaraki 305-0801, Japan

E-mail: v-itoudtokuyama.ac.jp

\section{Jun Nishimura}

KEK Theory Center, High Energy Accelerator Research Organization, 1-1 Oho, Tsukuba, Ibaraki 305-0801, Japan and Graduate University for Advanced Studies (SOKENDAI), 1-1 Oho, Tsukuba, Ibaraki 305-0801, Japan

E-mail: jnishidpost.kek.jp

\section{Toshiyuki Okubo}

Faculty of Science and Technology, Meijo University, Nagoya, 468-8502, Japan

E-mail: tokubodmeijo-u.ac.jp

\section{Stratos Kovalkov Papadoudis*}

Physics Department, National Technical University of Athens, Zografou Campus, GR-15780

Zografou, Greece

E-mail: sp10018dcentra1.ntua.qr 
The IKKT matrix model has been conjectured to provide a promising nonperturbative formulation of superstring theory. In this model, spacetime emerges dynamically from the microscopic matrix degrees of freedom in the large- $N$ limit, and Monte Carlo simulations of the Lorentzian version provide evidence of an emergent (3+1)-dimensional expanding space-time. In this talk, we discuss the Euclidean version of the IKKT matrix model and provide evidence of dynamical compactification of the extra dimensions via the spontaneous symmetry breaking (SSB) of the 10D rotational symmetry. We perform numerical simulations of a system with a severe complex action problem by using the complex Langevin method (CLM). The CLM suffers from the singular-drift problem and we deform the model in order to avoid it. We study the SSB pattern as we vary the deformation parameter and we conclude that the original model has an $\mathrm{SO}(3)$ symmetric vacuum, in agreement with previous calculations using the Gaussian expansion method (GEM). We employ the GEM to the deformed model and we obtain results consistent with the ones obtained by CLM.

Corfu Summer Institute 2019 "School and Workshops on Elementary Particle Physics and Gravity" (CORFU2019)

31 August - 25 September 2019

Corfu, Greece

${ }^{*}$ Speaker. 


\section{Introduction}

The type IIB matrix model [四, also known as the IKKT model, has been proposed as a nonperturbative formulation of superstring theory. Spacetime emerges dynamically from the eigenvalues of the bosonic matrices in the large- $N$ limit [[] and it is possible that the extra dimensions are compactified dynamically via a non perturbative mechanism. Furthermore, it is possible that a unique vacuum exists in the theory, thereby solving the so-called landscape problem.

The action of the model can be formally viewed as the dimensional reduction of the $10 \mathrm{D}$, $\mathscr{N}=1, \mathrm{SU}(N)$ super Yang-Mills (SYM) theory to zero dimensions. Numerical simulations [ [ 3 , $\mathbb{A}$,

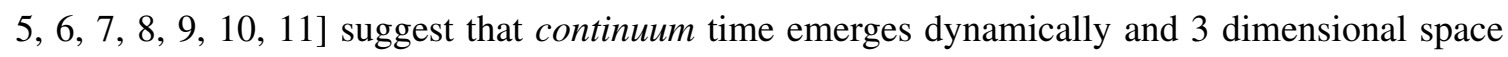
undergoes rapid expansion after a critical time $t_{c}$, while the remaining 6 dimensions do not expand. The cosmological time is defined by the eigenvalues of the temporal matrix $A_{0}$, and their infinite and homogeneous distribution in the large $N$ limit is a nontrivial dynamic result, allowing one to define a continuum and infinitely extending time. Furthermore, the spatial matrices $A_{i}$ have a band diagonal structure in the $\mathrm{SU}(N)$ basis used to diagonalize $A_{0}$ that makes possible to define space at a given time. Then one can study the structure of space, and especially its size, which for $t>t_{c}$, it is found to be expanding in the three dominant dimensions. This expansion is exponential at early times [5] and becomes a power law at later times [[]], making possible the emergence of a realistic cosmology from the dynamics of the microscopic degrees of freedom.

Simulations of the model using Monte Carlo techniques is hard because of the complex action problem. This originates from the $\mathrm{e}^{i S_{\mathrm{b}}}$ factor in the path integral, where $S_{\mathrm{b}}$ is the bosonic part of the action. This problem can be avoided by integrating the scale factor of the bosonic matrices

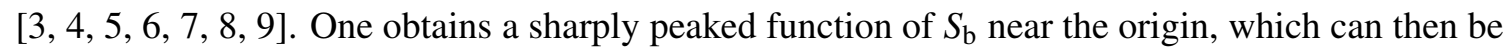
approximated by a Gaussian. The complex action problem vanishes, but the approximation favors

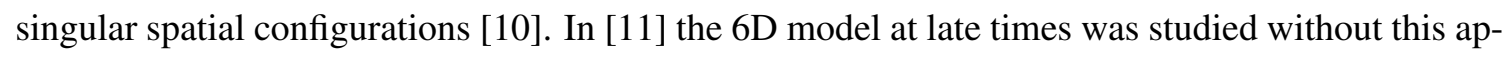
proximation, and the Complex Langevin Method (CLM) [12, [13] was used in order to confront the complex action problem. A two parameter deformation of the model was used for making the simulations possible, and provided evidence that when these parameters become small, configurations with non trivial spatial structure dominate the path integral.

In this work we study the Euclidean version of the IKKT matrix model. This model, as well as

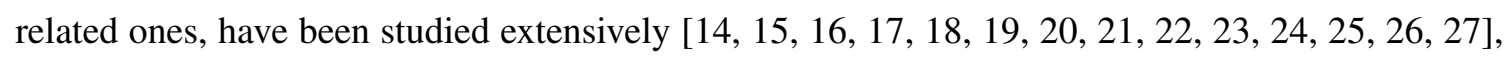
because they are more tractable numerically and are finite [대, [6], making the introduction of infrared cutoffs unnecessary. There is strong evidence that dynamical compactification of extra dimensions in these models is realized via spontaneous symmetry breaking (SSB) of the $\mathrm{SO}(10)$ rotational symmetry of the model. The effective action of the model $S_{\text {eff }}=S_{\mathrm{R}}+i \Gamma$, obtained after integrating out the fermionic degrees of freedom, is complex. The fluctuations of the phase $\mathrm{e}^{i \Gamma}$ for a generic $\mathrm{SO}(d), d>3$ symmetric configuration, suppress $d>3$ configurations, thereby favoring lower dimensional configurations and triggering the SSB [[28]. The evidence is provided mainly by studying the model using the Gaussian Expansion Method (GEM). In [29, BO, B], B2], B3], it was shown that the $\mathrm{SO}(3)$ vacuum of the IIB matrix model has the lowest free energy and the ratio between the extended three directions and the shrunken seven directions is a finite number, larger than one. Furthermore, the study of phase quenched models, show no SSB of the SO(10) rotational symmetry [패, [1], [8, [1]], consistent with the expectation that the complex phase $\mathrm{e}^{i \Gamma}$ 
plays a critical role in suppressing large dimensional configurations.

The Monte Carlo simulations of the Euclidean IIB matrix model confront a very strong complex action problem, which needs to be addressed by using special methods. Straightforward reweighting is not possible, since it makes the computational effort to increase exponentially with the matrix size $N$, which needs to be extrapolated to infinity. A density of states based method was used in [201, [34, [35, 22], 223, 24, 25], allowing one to study relatively large systems and providing evidence that SSB occurs from first principles. But it turned out to be hard to determine the pattern of SSB, and it was not until recently [26, [36, [27] that this question could be addressed by using the Complex Langevin Method (CLM)[12], [3], [3]]. The CLM is applied by complexifying the degrees of freedom and defining a stochastic process where the expectation values with respect to this process are equal to the expectation values defined in the original system. This method fails in many cases, and it was not until recently that with the help of new techniques [38, 139, 40, 4], 42] it was possible to meet the conditions for the equivalence of the stochastic process defined by the CLM and the original system, and obtain correct results for an extended range of parameters [43], 44, 45, [21, 16, 47, 18]]. The CLM has been applied successfully to many systems in lattice quantum field

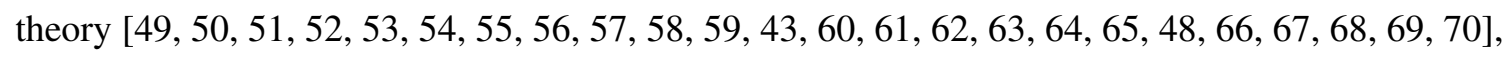

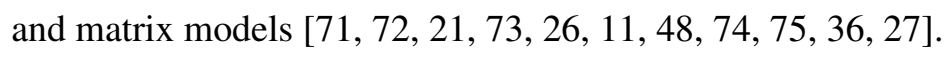

In this talk we review the application of the CLM to the Euclidean type IIB matrix model discussed in [26, [36, 27]. The successful application of the method requires the deformation of the model by two parameters $m_{\mathrm{f}}$ and $\varepsilon$, which deform the Dirac operator and the Bosonic part of the action with mass like terms. This way, one can avoid the singular drift problem [ [D]], which can lead to erroneous results in systems where singularities of the drift dominate in the stochastic process of the CLM [40]. In the IIB matrix model the singular drift problem occurs when the eigenvalues of the Pfaffian, when put into a canonical Youla's form, accumulate near zero. This method was applied successfully on the 6D IIB model [26], obtaining SSB to SO(3), consistent with GEM calculations in [32]. The 10D model was studied in [27]. In that case, the simulations were harder because the fermionic degrees of freedom increase by a factor of four and the finite size effects are more severe due to the increase of the dimensionality of the target space. The results are consistent with GEM calculations [B3], showing SSB to $\mathrm{SO}(3)$. A careful extrapolation of the results, first to large $N$, then to small $\varepsilon$ and finally to small $m_{\mathrm{f}}$ is necessary in order to obtain the correct large $N$ limit of the original model. This makes the calculation tricky, and the Monte Carlo simulations were done in parallel with GEM calculations of the $m_{\mathrm{f}}$-deformed model. By computing the free energy of vacua of different dimensionality using the GEM, it was possible to find physical solutions for the $\mathrm{SO}(d), d=4,6,7$ ansatzes, calculated up to three loops. As $m_{\mathrm{f}}$ decreases, lower dimensional vacua turn out to have lower free energies and the pattern of SSB is similar to the one obtained by the CLM. 


\section{The IKKT matrix model}

The IKKT matrix model [四] is defined by the action:

$$
\begin{aligned}
S & =S_{\mathrm{b}}+S_{\mathrm{f}}, \text { where } \\
S_{\mathrm{b}} & =-\frac{1}{4} N \operatorname{tr}\left[A_{\mu}, A_{v}\right]\left[A^{\mu}, A^{v}\right], \\
S_{\mathrm{f}} & =-\frac{1}{2} N \operatorname{tr}\left(\psi_{\alpha}\left(\mathscr{C} \Gamma^{\mu}\right)_{\alpha \beta}\left[A_{\mu}, \psi_{\beta}\right]\right) .
\end{aligned}
$$

For $D=10$, the matrices $A_{\mu}(\mu=0,1,2, \ldots, D-1)$ are $N \times N$ traceless Hermitian matrices which transform like vectors, and $\psi_{\alpha}\left(\alpha=1,2, \ldots, 2^{D / 2-1}\right)$ are $N \times N$ traceless matrices with Grassmann entries which transform like Majorana-Weyl spinors. The $2^{D / 2-1} \times 2^{D / 2-1}$ matrices $\Gamma^{\mu}$ and $\mathscr{C}$ are the gamma matrices after Weyl projection and the charge conjugation matrix, respectively, in ten dimensions.

In order to obtain the Euclidean version of the IKKT matrix model, we perform the Wick rotation

$$
A_{0}=i A_{D}, \quad \Gamma^{0}=-i \Gamma^{D}
$$

The metric now is $\delta_{\mu v}(\mu, v=1, \ldots, D)$ and the partition function becomes

$$
Z=\int d A d \psi e^{-S}=\int d A e^{-S_{\mathrm{b}}} \operatorname{Pf} \mathscr{M}
$$

where the $2^{D / 2-1}\left(N^{2}-1\right) \times 2^{D / 2-1}\left(N^{2}-1\right)$ anti-symmetric matrix $\mathscr{M}$ is defined by its action

$$
\psi_{\alpha} \rightarrow(\mathscr{M} \psi)_{\alpha}=\left(\mathscr{C} \Gamma^{\mu}\right)_{\alpha \beta}\left[A_{\mu}, \psi_{\beta}\right]
$$

on the linear space of traceless complex $N \times N$ matrices. The model has an $\mathrm{SO}(D)$ rotational symmetry acting on $A_{\mu}$ and $\psi_{\alpha}$.

Dynamical compactification of extra dimensions can be realized via the SSB of the $\mathrm{SO}(D)$ symmetry to $\mathrm{SO}(d)$, with $d<D$. The order parameters of the SSB can be taken to be [2], 26]

$$
\lambda_{\mu}=\frac{1}{N} \operatorname{tr}\left(A_{\mu}\right)^{2}, \quad \mu=1, \ldots, D,
$$

where no sum over $\mu$ is taken. Then, we break the $\mathrm{SO}(D)$ symmetry explicitly by deforming the model by

$$
\Delta S_{\mathrm{b}}=\frac{1}{2} N \varepsilon \sum_{\mu=1}^{D} m_{\mu} \operatorname{tr}\left(A_{\mu}\right)^{2}
$$

using the parameters $\varepsilon$ and $0<m_{1} \leq \ldots \leq m_{D}$. The SSB pattern will arise in the $\varepsilon \rightarrow 0$ limit after taking the large- $N$ limit. For finite $N$, the choice $0<m_{1} \leq \ldots \leq m_{D}$ yields $\left\langle\lambda_{1}\right\rangle \geq \ldots \geq\left\langle\lambda_{D}\right\rangle$. If there is no SSB, all $\left\langle\lambda_{\mu}\right\rangle$ will be equal in the $N \rightarrow \infty$ and $\varepsilon \rightarrow 0$ limits. Otherwise, SSB occurs and we will conclude that some dimensions of space are larger than others.

The SSB of the $\mathrm{SO}(D)$ rotational symmetry has been studied by using the GEM. This method uses a systematic expansion around a Gaussian action $S_{0}$, which contains many parameters. In order to make the calculation tractable, the number of parameters is reduced by considering $\mathrm{SO}(d)-$ symmetric ansatzes for $d<D$. The free energy and the expectation values of the observables is 
calculated in the expansion around $S_{0}$ as a function of these parameters, and the physical solutions are computed by finding a region in the parameter space where the results are independent of these parameters. In this way, one obtains nonperturbative information about the model [06]. When

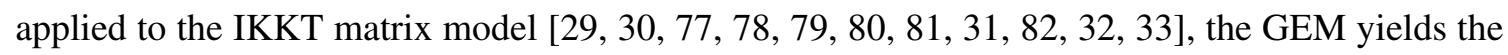
free energy and the average extent of spacetime in each direction. The $D=6$ case was studied in [B2], and free energy and the average extent of spacetime was calculated to fifth order. The $\mathrm{SO}(3)$ ansatz was found to have the lowest free energy, showing SSB to SO(3). The extended directions where found to have an extent $R^{2}(d)$ for $\mu=1, \ldots, d$, whereas the shrunken directions to have and extent $r^{2}=\left\langle\lambda_{\mu}\right\rangle \approx 0.223, \mu=d+1, \ldots, D$, which is independent of $d$. The values of $R^{2}(d)$ are such that

$$
R^{d}(d) r^{D-d} \approx l^{D}
$$

where $v \equiv l^{D}$ is the spacetime volume. It turns out that $l^{2} \approx 0.627$ and $R^{2}(3) \approx 1.76$. The $D=10$ case was studied in [33], where a systematic expansion to third order was performed for $\operatorname{SO}(d)$ ansatzes with $2 \leq d \leq 7$. The $\mathrm{SO}(3)$ ansatz turns out to have the lowest free energy, also showing SSB to $\mathrm{SO}(3)$. In that case

$$
R^{2}(3) \approx 3.27, \quad r^{2} \approx 0.155, \quad l^{2} \approx 0.383 .
$$

These results are consistent with the ones obtained by Monte Carlo simulations [25]. They are finite, in contrast to the case of the Lorentzian model, where $R^{2}(d)$ expands indefinitely.

\section{The Complex Langevin Method}

In this section we review how to apply the CLM to the Euclidean IKKT matrix model. The partition function can be written as

$$
Z=\int d A \mathrm{e}^{-S_{\text {eff }}},
$$

where the effective action $S_{\text {eff }}=S_{\mathrm{b}}-\log \operatorname{Pf} \mathscr{M}$ is complex. In the CLM the matrices $A_{\mu}$ become general complex traceless matrices, which amounts to the complexification of the degrees of freedom in the CLM. The time evolution in the fictitious Langevin time is given by

$$
\frac{d\left(A_{\mu}(t)\right)_{i j}}{d t}=-\frac{\partial S_{\mathrm{eff}}\left[A_{\mu}(t)\right]}{\partial\left(A_{\mu}\right)_{j i}}+\left(\eta_{\mu}\right)_{i j}(t) .
$$

The noise $\eta_{\mu}(t)$ is made from traceless Hermitian matrices whose elements are random variables obeying the Gaussian distribution $\propto \exp \left(-\frac{1}{4} \int \operatorname{tr}\left\{\eta_{\mu}(t)\right\}^{2} d t\right)$. The first term on the right-hand side is the drift term

$$
\frac{\partial S_{\mathrm{eff}}}{\partial\left(A_{\mu}\right)_{j i}}=\frac{\partial S_{\mathrm{b}}}{\partial\left(A_{\mu}\right)_{j i}}-\frac{1}{2} \operatorname{Tr}\left(\mathscr{M}^{-1} \frac{\partial \mathscr{M}}{\partial\left(A_{\mu}\right)_{j i}}\right),
$$

where $\operatorname{Tr}$ represents the trace of a $16\left(N^{2}-1\right) \times 16\left(N^{2}-1\right)$ matrix. The expectation value of an observable $\mathscr{O}\left[A_{\mu}\right]$ can be calculated from

$$
\left\langle\mathscr{O}\left[A_{\mu}\right]\right\rangle=\frac{1}{T} \int_{t_{0}}^{t_{0}+T} \mathscr{O}\left[A_{\mu}(t)\right] d t,
$$


where $A_{\mu}(t)$ is a general complex matrix solution of (B.2), $t_{0}$ is the thermalization time, and $T$ is large enough in order to obtain good statistics. Upon complexification of the matrices $A_{\mu}(t)$, the observable $\mathscr{O}\left[A_{\mu}(t)\right]$ depends on general complex matrices. The analyticity of the function $\mathscr{O}\left[A_{\mu}\right]$ plays a crucial role in the proof of the validity of (B.4) [B8, B. $3 ., 4]$. The discretization of (B.2) is given by

$$
\left(A_{\mu}\right)_{i j}(t+\Delta t)=\left(A_{\mu}\right)_{i j}(t)-\Delta t \frac{\partial S\left[A_{\mu}(t)\right]}{\partial\left(A_{\mu}\right)_{j i}}+\sqrt{\Delta t}\left(\eta_{\mu}\right)_{i j}(t),
$$

which we have used in order to solve (B.2) numerically.

The solutions of the stochastic equation (B.2) are random variables with distribution $P\left(A_{\mu}^{(\mathrm{R})}, A_{\mu}^{(\mathrm{I})} ; t\right)$, where $A_{\mu}^{(\mathrm{R})}(t)=\left(A_{\mu}(t)+A_{\mu}^{\dagger}(t)\right) / 2, A_{\mu}^{(\mathrm{I})}(t)=\left(A_{\mu}(t)-A_{\mu}^{\dagger}(t)\right) / 2 i$, and in order that in the $t \rightarrow \infty$ limit an observable $\mathscr{O}\left[A_{\mu}\right]$ has an expectation value in this distribution equal to $\left\langle\mathscr{O}\left[A_{\mu}\right]\right\rangle$, given by the path integral $(2.5)$, certain conditions need to be met. In [4] it was shown that if the magnitude of the drift falls off exponentially or faster, then these conditions are met. For this, the $A_{\mu}(t)$ should not make long trips in the anti-Hermitian direction. Gauge cooling has been applied in order to restrict those trips and meet this condition[26, 27]. Adaptive stepsize techniques have also been applied in order to keep the stability of the time evolution. Furthermore, one has to avoid the singular drift problem. This problem occurs in the term with $\mathscr{M}^{-1}$ in Eq. (B.3]), when the eigenvalues of $\mathscr{M}$ accumulate densely near zero. In order to avoid this problem, we deform the fermionic action by adding a term

$$
\Delta S_{\mathrm{f}}=-i m_{\mathrm{f}} \frac{N}{2} \operatorname{tr}\left(\psi_{\alpha}\left(\mathscr{C} \Gamma_{8} \Gamma_{9}^{\dagger} \Gamma_{10}\right)_{\alpha \beta} \psi_{\beta}\right)
$$

where $m_{\mathrm{f}}$ is the deformation parameter. This term shifts the eigenvalue distribution of $\mathscr{M}$ away from the origin [2]], but it also breaks the $\mathrm{SO}(10)$ symmetry down to $\mathrm{SO}(7) \times \mathrm{SO}(3)$ explicitly. Therefore, we examine if the remaining $\mathrm{SO}(7)$ symmetry breaks down to smaller subgroups as $m_{\mathrm{f}}$ is varied and discuss what occurs at $m_{\mathrm{f}}=0$. As $m_{\mathrm{f}} \rightarrow \infty$, the fermionic degrees of freedom decouple and we obtained the so-called bosonic model. This model is known to be $\mathrm{SO}(10)$ symmetric and no SSB occurs [25].

The severeness of the singular drift problem depends on the parameters $m_{\mathrm{f}}$ and $\varepsilon$. For large enough $m_{\mathrm{f}}$ the problem disappears, but as $m_{\mathrm{f}}$ is lowered the problem reappears for small enough $\varepsilon$. In our simulations we monitor the fall off of the drift and make sure that it falls of faster than exponentially. The singular drift problem is expected to vanish for large enough $N$ [27].

\section{Results}

In our numerical investigation we consider whether the remaining $\mathrm{SO}(7)$ symmetry is broken down to a smaller group. We study the model deformed by (‥8) and (B.6). We use $m_{\mathrm{f}}=3.0$, 1.4, 1.0, 0.9, 0.7, in order to extrapolate to the undeformed IKKT model at $m_{\mathrm{f}}=0$. The $m_{\mu}$ in Eq. (2.8) are chosen so that this term does not break $\mathrm{SO}(10)$ completely, because otherwise the spectrum of $m_{\mu}$ becomes too wide to make the $\varepsilon \rightarrow 0$ extrapolation reliably. For $m_{\mathrm{f}}=3.0$, we choose $m_{\mu}=(0.5,0.5,0.5,1,2,4,8,8,8,8)$, which enables us to distinguish $\mathrm{SO}(d)$ vacua with $d=3,4,5,6,7$. For smaller values of $m_{\mathrm{f}}$, we choose $m_{\mu}=(0.5,0.5,1,2,4,8,8,8,8,8)$, which enables us to distinguish $\mathrm{SO}(d)$ vacua with $d=2,3,4,7$. In particular, we may confirm that the $\mathrm{SO}(3)$ symmetry remains unbroken by seeing that $\left\langle\lambda_{1}\right\rangle=\left\langle\lambda_{2}\right\rangle$ and $\left\langle\lambda_{3}\right\rangle$ agree in the $N \rightarrow \infty$ and 
$\varepsilon \rightarrow 0$ limits. On the other hand, this choice of $m_{\mu}$ has a drawback that $\lambda_{6}$ and $\lambda_{7}$ are mixed up because of $m_{6}=m_{7}$, and hence one cannot distinguish $\mathrm{SO}(5)$ and $\mathrm{SO}(6)$ vacua. This does not cause any harm, however, as far as we find that $\left\langle\lambda_{4}\right\rangle$ and $\left\langle\lambda_{5}\right\rangle$ do not agree in the $N \rightarrow \infty$ and $\varepsilon \rightarrow 0$ limits, which implies that the $\mathrm{SO}(7)$ symmetry is broken to $\mathrm{SO}(4)$ or lower symmetries.
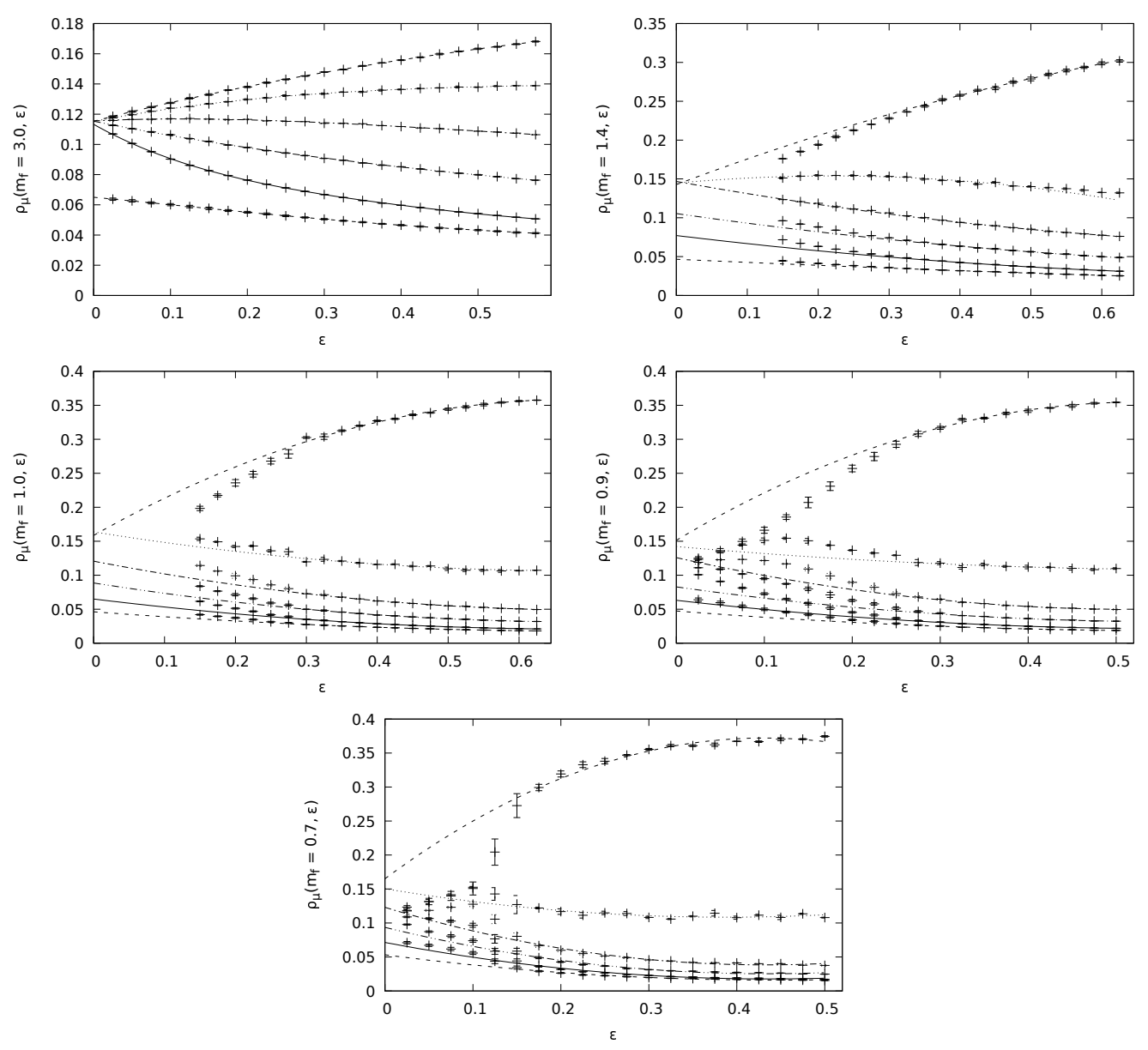

Figure 1: The $\rho_{\mu}\left(m_{\mathrm{f}}, \varepsilon\right)$ in Eq. (4.2) are plotted against $\varepsilon$ for $m_{\mathrm{f}}=3.0$ (Top-Left), $m_{\mathrm{f}}=1.4$ (TopRight), $m_{\mathrm{f}}=1.0$ (Middle-Left), $m_{\mathrm{f}}=0.9$ (Middle-Right) and $m_{\mathrm{f}}=0.7$ (Bottom). We use $m_{\mu}=$ $(0.5,0.5,0.5,1,2,4,8,8,8,8)$ for $m_{\mathrm{f}}=3.0$ and $m_{\mu}=(0.5,0.5,1,2,4,8,8,8,8,8)$ for the other values of $m_{\mathrm{f}}$. The continuous lines are polynomial fits in $\varepsilon$. For $m_{\mathrm{f}}=3.0$ a quartic fit is performed, whereas for the other values of $m_{\mathrm{f}}$ the fits are quadratic in $\varepsilon$. In the $m_{\mathrm{f}}=3.0$ plot, the curves from top to bottom are $\left(\rho_{1}+\rho_{2}+\rho_{3}\right) / 3, \rho_{4}, \rho_{5}, \rho_{6}, \rho_{7}$ and $\left(\rho_{8}+\rho_{9}+\rho_{10}\right) / 3$. For the other plots, the curves from top to bottom are $\left(\rho_{1}+\rho_{2}\right) / 2, \rho_{3}, \rho_{4}, \rho_{5},\left(\rho_{6}+\rho_{7}\right) / 2$ and $\left(\rho_{8}+\rho_{9}+\rho_{10}\right) / 3$.

In order to probe the SSB, one has to take the $N \rightarrow \infty$ limit first and then the $\varepsilon \rightarrow 0$ limit. For given $m_{\mu}$ in Eq. ( $(2.8)$, the large- $N$ limit is obtained by first computing the ratio

$$
\rho_{\mu}\left(m_{\mathrm{f}}, \varepsilon, N\right)=\frac{\left\langle\lambda_{\mu}\right\rangle_{m_{\mathrm{f}}, \varepsilon, N}}{\sum_{v=1}^{10}\left\langle\lambda_{v}\right\rangle_{m_{\mathrm{f}}, \varepsilon, N}}
$$

and then by making a large- $N$ extrapolation

$$
\rho_{\mu}\left(m_{\mathrm{f}}, \mathcal{\varepsilon}\right)=\lim _{N \rightarrow \infty} \rho_{\mu}\left(m_{\mathrm{f}}, \mathcal{\varepsilon}, N\right)
$$


The large- $N$ extrapolation is performed by plotting $\rho_{\mu}\left(m_{\mathrm{f}}, \varepsilon, N\right)$ against $1 / N$ and making a quadratic fit with respect to $1 / N$. Then we make the $\varepsilon \rightarrow 0$ extrapolation

$$
\rho_{\mu}\left(m_{\mathrm{f}}\right)=\lim _{\varepsilon \rightarrow 0} \rho_{\mu}\left(m_{\mathrm{f}}, \varepsilon\right)
$$

by fitting $\rho_{\mu}\left(m_{\mathrm{f}}, \varepsilon\right)$ to a polynomial in $\varepsilon$. In Fig. Wwe plot the large- $N$ extrapolated values $\rho_{\mu}\left(m_{\mathrm{f}}, \varepsilon\right)$ as a function of $\varepsilon$ for $m_{\mathrm{f}}=3.0,1.4,1.0,0.9$ and 0.7 together with the performed fits. In those fits, the data that is affected by finite size effects must be excluded. As it was argued in [27], these become quite severe in the $\varepsilon \rightarrow 0$ limit. These effects are apparent in Fig. $\mathbb{W}$, where a crossover to a symmetric phase is observed for $m_{\mathrm{f}} \leq 1.4$ as $\varepsilon \rightarrow 0$. This crossover is expected to vanish in the large- $N$ limit.

From the extrapolated values $\rho_{\mu}\left(m_{\mathrm{f}}\right)$, we find that the $\mathrm{SO}(7)$ symmetry of the deformed model is not spontaneously broken at $m_{\mathrm{f}}=3.0$, but it is actually broken to $\mathrm{SO}(4)$ for $m_{\mathrm{f}}=1.4$ and to $\mathrm{SO}(3)$ for $m_{\mathrm{f}}=1.0,0.9,0.7$. Thus as $m_{\mathrm{f}}$ is decreased, the $\mathrm{SO}(7)$ symmetry seems to be spontaneously broken to smaller subgroups gradually in the same way as it was observed in the $D=6$ case [26]. However, we consider that the symmetry is not going to be broken further down to $\mathrm{SO}(2)$ at smaller $m_{\mathrm{f}}$. This is based on the fact that the Pfaffian vanishes identically for strictly $2 \mathrm{D}$ configurations [28, [83], which implies that the mechanism of SSB due to the phase of the Pfaffian no longer works there ${ }^{1}$. Hence our results are consistent with the results obtained by the GEM for the undeformed model, which show that the $\mathrm{SO}(3)$ vacuum has the smallest free energy.

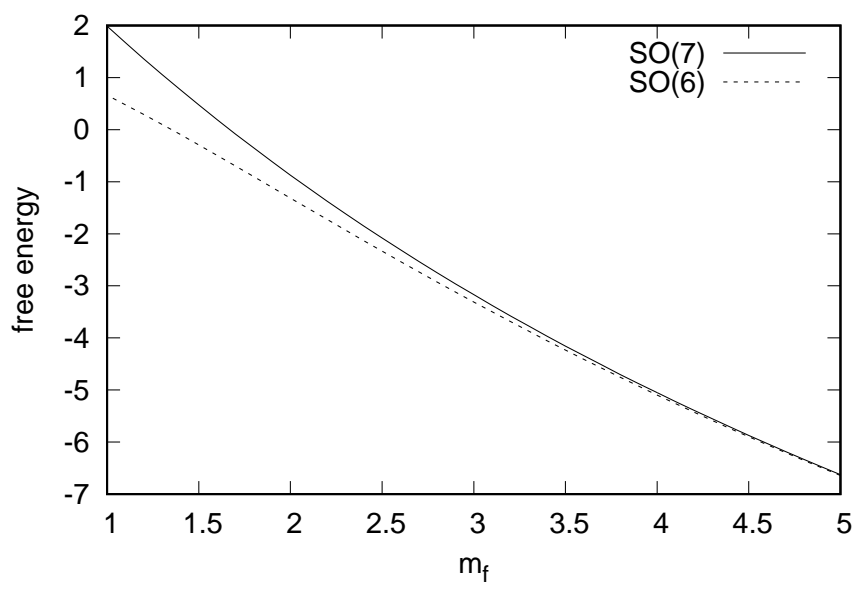

Figure 2: The free energy calculated up to three loops for the solutions found with the $\operatorname{SO}(7)$ and $\mathrm{SO}(6)$ ansatzes are plotted against the fermion mass $m_{\mathrm{f}}$. We observe a clear tendency that the $\mathrm{SO}(6)$ symmetric vacuum is more favored as $m_{\mathrm{f}}$ is decreased, whereas the free energy for the two ansatzes tends to be degenerate as $m_{\mathrm{f}}$ is increased.

The above mentioned results have also been checked against a calculation using the GEM on the deformed model (B.6). The GEM has been applied to the Euclidean IKKT matrix model in [B3], where $\mathrm{SO}(10)$ was found to be broken down to SO(3). The GEM has the advantage that the

\footnotetext{
${ }^{1}$ This is also reflected in the GEM results [B2, [33] for the free energy of the $\mathrm{SO}(d)$ vacuum, which becomes much larger for $d=2$ than for $d \geq 3$.
} 
large- $N$ limit is easily taken by considering only planar graphs and that the small- $\varepsilon$ extrapolation is not necessary. The systematic errors of the GEM are due to the truncation of the expansion and the errors in determining the parameters that give the physical solutions. As such, the two methods can be considered to be completely independent. In [2]], we performed a three-loop calculation using $\mathrm{SO}(d)$ symmetric ansatzes for $d=6,7$, and calculated the free energy. We observed that by decreasing $m_{\mathrm{f}}$, the free energy of the $\mathrm{SO}(6)$ vacuum becomes smaller than the free energy of the SO(7) vacuum. In Fig. \ we plot the free energy calculated up to three loops for the solutions found with the $\mathrm{SO}(7)$ and $\mathrm{SO}(6)$ ansatzes against the fermion mass $m_{\mathrm{f}}$. We observe a clear tendency that the $\mathrm{SO}(6)$ symmetric vacuum is more favored as $m_{\mathrm{f}}$ is decreased. However, the free energy for the two ansatzes tends to become degenerate as $m_{\mathrm{f}}$ is increased. In this situation it is difficult to identify the critical point, given the accuracy of the GEM results.

At $m_{\mathrm{f}}=3.0$, the extent of space was found to agree very well between the two methods. In [D]], it is found that

$$
\rho_{1}=\cdots=\rho_{7}=0.116, \quad \rho_{8}=\rho_{9}=\rho_{10}=0.064,
$$

whereas the CLM results of Fig. $⿴$ give

$$
\rho_{1}=\cdots=\rho_{7}=0.115, \quad\left(\rho_{8}+\rho_{9}+\rho_{10}\right) / 3=0.065 .
$$

Therefore, for the first time, we have a first principle study of the Euclidean IKKT matrix model that produced clear results on the question of dynamical compactification of extra dimensions via SSB of the SO(10) rotational symmetry of the model. The $\mathrm{SO}(10)$ rotational symmetry breaking of the Euclidean IKKT matrix model down to $\mathrm{SO}(3)$ due to the phase of the Pfaffian is interesting, but it makes the model somewhat difficult to interpret. Given the promising properties

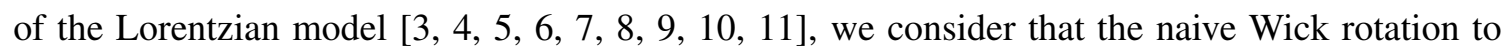
the Euclidean model is not the right direction to pursue. On the other hand, the fact that the CLM enabled us to obtain a clear SSB pattern for the deformed model, which suffers from a severe sign problem, is encouraging. We hope that the CLM is equally useful in investigating the Lorentzian IKKT model, in particular in the presence of fermionic matrices, which are not included yet in Ref. [U]].

\section{Acknowledgements}

The authors would like to thank S. Iso, H. Kawai, H. Steinacker and A. Tsuchiya for valuable discussions. T. A. was supported in part by Grant-in-Aid for Scientific Research (No. 17K05425) from Japan Society for the Promotion of Science. Computations were carried out using computational facilities at KEKCC and the NTUA het cluster. This work was also supported by computational time granted by the Greek Research \& Technology Network (GRNET) in the National HPC facility - ARIS - under project ID IKKT10D.

\section{References}

[1] N. Ishibashi, H. Kawai, Y. Kitazawa, and A. Tsuchiya, A large N reduced model as superstring, Nucl. Phys. B498 (1997) 467-491, [hep-th/9612115]. 
[2] H. Aoki, S. Iso, H. Kawai, Y. Kitazawa, and T. Tada, Space-time structures from IIB matrix model, Prog. Theor. Phys. 99 (1998) 713-746, [hep-th/9802085].

[3] S.-W. Kim, J. Nishimura, and A. Tsuchiya, Expanding (3+1)-dimensional universe from a Lorentzian matrix model for superstring theory in (9+1)-dimensions, Phys. Rev. Lett. 108 (2012) 011601 , [arXiv:1108,1540].

[4] Y. Ito, J. Nishimura, and A. Tsuchiya, Universality and the dynamical space-time dimensionality in the Lorentzian type IIB matrix model, JHEP 03 (2017) 143, [arXiv: 1701.07783].

[5] Y. Ito, S.-W. Kim, Y. Koizuka, J. Nishimura, and A. Tsuchiya, A renormalization group method for studying the early universe in the Lorentzian IIB matrix model, PTEP 2014 (2014), no. 8 083B01, [arXiv:1312.5415].

[6] Y. Ito, S.-W. Kim, J. Nishimura, and A. Tsuchiya, Monte Carlo studies on the expanding behavior of the early universe in the Lorentzian type IIB matrix model, PoS LATTICE2013 (2014) 341, [arxiv:13]1.5579].

[7] Y. Ito, J. Nishimura, and A. Tsuchiya, Large-scale computation of the exponentially expanding universe in a simplified Lorentzian type IIB matrix model, PoS LATTICE2015 (2016) 243, [arXiv:1512.01923].

[8] Y. Ito, J. Nishimura, and A. Tsuchiya, Power-law expansion of the Universe from the bosonic Lorentzian type IIB matrix model, JHEP 11 (2015) 070, [arXiv: 1506.04795].

[9] T. Azuma, Y. Ito, J. Nishimura, and A. Tsuchiya, A new method for probing the late-time dynamics in the Lorentzian type IIB matrix model, PTEP 2017 (2017), no. 8 083B03, [arXiv:1705.07812].

[10] T. Aoki, M. Hirasawa, Y. Ito, J. Nishimura, and A. Tsuchiya, On the structure of the emergent $3 d$ expanding space in the Lorentzian type IIB matrix model, PTEP 2019 (2019), no. 9 093B03, [arxiv: [904.05914].

[11] J. Nishimura and A. Tsuchiya, Complex Langevin analysis of the space-time structure in the Lorentzian type IIB matrix model, JHEP 06 (2019) 077, [arxiv:1904.05919].

[12] G. Parisi, On Complex Probabilities, Phys. Lett. B131 (1983) 393-395.

[13] J. R. Klauder, Coherent State Langevin Equations for Canonical Quantum Systems With Applications to the Quantized Hall Effect, Phys. Rev. A29 (1984) 2036-2047.

[14] T. Hotta, J. Nishimura, and A. Tsuchiya, Dynamical aspects of large N reduced models, Nucl. Phys. B545 (1999) 543-575, [hep-th/9811220].

[15] P. Austing and J. F. Wheater, The Convergence of Yang-Mills integrals, JHEP 02 (2001) 028, [hep-th/010107]].

[16] P. Austing and J. F. Wheater, Convergent Yang-Mills matrix theories, JHEP 04 (2001) 019, [hep-th/0103159].

[17] J. Ambjorn, K. N. Anagnostopoulos, W. Bietenholz, T. Hotta, and J. Nishimura, Large N dynamics of dimensionally reduced 4-D SU(N) superYang-Mills theory, JHEP 07 (2000) 013, [hep-th/0003208].

[18] J. Ambjorn, K. N. Anagnostopoulos, W. Bietenholz, T. Hotta, and J. Nishimura, Monte Carlo studies of the IIB matrix model at large N, JHEP 07 (2000) 011, [hep-th/0005147].

[19] J. Ambjorn, K. N. Anagnostopoulos, W. Bietenholz, F. Hofheinz, and J. Nishimura, On the spontaneous breakdown of Lorentz symmetry in matrix models of superstrings, Phys. Rev. D65 (2002) 086001, [hep-th/0104260]. 
[20] K. N. Anagnostopoulos and J. Nishimura, New approach to the complex action problem and its application to a nonperturbative study of superstring theory, Phys. Rev. D66 (2002) 106008, [hep-th/010804]].

[21] Y. Ito and J. Nishimura, The complex Langevin analysis of spontaneous symmetry breaking induced by complex fermion determinant, JHEP 12 (2016) 009,

[22] K. N. Anagnostopoulos, T. Azuma, and J. Nishimura, A general approach to the sign problem: The factorization method with multiple observables, Phys. Rev. D83 (2011) 054504, [arxiv:1009.4504].

[23] K. N. Anagnostopoulos, T. Azuma, and J. Nishimura, A practical solution to the sign problem in a matrix model for dynamical compactification, JHEP 10 (2011) 126, [arXiv:1108.1534].

[24] K. N. Anagnostopoulos, T. Azuma, and J. Nishimura, Monte Carlo studies of the spontaneous rotational symmetry breaking in dimensionally reduced super Yang-Mills models, JHEP 11 (2013) 009, [arXiv:1306.6135].

[25] K. N. Anagnostopoulos, T. Azuma, and J. Nishimura, Monte Carlo studies of dynamical compactification of extra dimensions in a model of nonperturbative string theory, PoS LATTICE2015 (2016) 307, [arXiv:1509.05079].

[26] K. N. Anagnostopoulos, T. Azuma, Y. Ito, J. Nishimura, and S. K. Papadoudis, Complex Langevin analysis of the spontaneous symmetry breaking in dimensionally reduced super Yang-Mills models, JHEP 02 (2018) 151, [arXiv:1712.07562].

[27] K. N. Anagnostopoulos, T. Azuma, Y. Ito, J. Nishimura, T. Okubo, and S. Kovalkov Papadoudis, Complex Langevin analysis of the spontaneous breaking of $10 D$ rotational symmetry in the Euclidean IKKT matrix model, arXiv:2002.07410.

[28] J. Nishimura and G. Vernizzi, Spontaneous breakdown of Lorentz invariance in IIB matrix model, JHEP 04 (2000) 015, [hep-th/0003223].

[29] J. Nishimura and F. Sugino, Dynamical generation of four-dimensional space-time in the IIB matrix model, JHEP 05 (2002) 001, [hep-th/0111102].

[30] H. Kawai, S. Kawamoto, T. Kuroki, T. Matsuo, and S. Shinohara, Mean field approximation of IIB matrix model and emergence of four-dimensional space-time, Nucl. Phys. B647 (2002) 153-189, [hep-th/0204240].

[31] T. Aoyama and H. Kawai, Higher order terms of improved mean field approximation for IIB matrix model and emergence of four-dimensional space-time, Prog. Theor. Phys. 116 (2006) 405-415, [hep-th/0603146].

[32] T. Aoyama, J. Nishimura, and T. Okubo, Spontaneous breaking of the rotational symmetry in dimensionally reduced super Yang-Mills models, Prog. Theor. Phys. 125 (2011) 537-563, [arXiv:1007.0883].

[33] J. Nishimura, T. Okubo, and F. Sugino, Systematic study of the SO(10) symmetry breaking vacua in the matrix model for type IIB superstrings, JHEP 10 (2011) 135, [arxiv: 1108_1293].

[34] J. Ambjorn, K. N. Anagnostopoulos, J. Nishimura, and J. J. M. Verbaarschot, The factorization method for systems with a complex action: A test in random matrix theory for finite density $Q C D$, JHEP 10 (2002) 062, [hep-1at/0208025]. 
[35] J. Ambjorn, K. N. Anagnostopoulos, J. Nishimura, and J. J. M. Verbaarschot, Noncommutativity of the zero chemical potential limit and the thermodynamic limit in finite density systems, Phys. Rev. D70 (2004) 035010, [hep-1at/0402031].

[36] K. N. Anagnostopoulos, T. Azuma, Y. Ito, J. Nishimura, and S. K. Papadoudis, Dynamical compactification of extra dimensions in the Euclidean type IIB matrix model: A numerical study using the complex Langevin method, PoS CORFU2018 (2019) 065, [arXiv: 1906.0184]].

[37] G. Parisi and Y.-s. Wu, Perturbation Theory Without Gauge Fixing, Sci. Sin. 24 (1981) 483.

[38] G. Aarts, E. Seiler, and I.-O. Stamatescu, The Complex Langevin method: When can it be trusted?, Phys. Rev. D81 (2010) 054508, [arxiv:0912.3360].

[39] G. Aarts, F. A. James, E. Seiler, and I.-O. Stamatescu, Complex Langevin: Etiology and Diagnostics of its Main Problem, Eur. Phys. J. C71 (2011) 1756, [arxiv:1101.3270].

[40] J. Nishimura and S. Shimasaki, New insights into the problem with a singular drift term in the complex Langevin method, Phys. Rev. D92 (2015), no. 1 011501, [arXiv: 1504.08359].

[41] K. Nagata, J. Nishimura, and S. Shimasaki, Argument for justification of the complex Langevin method and the condition for correct convergence, Phys. Rev. D94 (2016), no. 11 114515, [arxiv: 1606.07627 ].

[42] L. L. Salcedo, Does the complex Langevin method give unbiased results?, Phys. Rev. D94 (2016), no. 11 114505, [arXiv:161].06390].

[43] E. Seiler, D. Sexty, and I.-O. Stamatescu, Gauge cooling in complex Langevin for QCD with heavy quarks, Phys. Lett. B723 (2013) 213-216, [arXiv:1211.3709].

[44] K. Nagata, J. Nishimura, and S. Shimasaki, Justification of the complex Langevin method with the gauge cooling procedure, PTEP 2016 (2016), no. 1 013B01, [arXiv: 1508.02377].

[45] S. Tsutsui and T. M. Doi, Improvement in complex Langevin dynamics from a view point of Lefschetz thimbles, Phys. Rev. D94 (2016), no. 7 074009, [arXiv:1508.04231].

[46] K. Nagata, J. Nishimura, and S. Shimasaki, Gauge cooling for the singular-drift problem in the complex Langevin method - a test in Random Matrix Theory for finite density QCD, JHEP 07 (2016) 073, [arXiv:1604.07717].

[47] T. M. Doi and S. Tsutsui, Modifying partition functions: a way to solve the sign problem, Phys. Rev. D96 (2017), no. 9 094511, [arxiv:1709.05806].

[48] K. Nagata, J. Nishimura, and S. Shimasaki, Testing the criterion for correct convergence in the complex Langevin method, JHEP 05 (2018) 004, [arXiv:1802.01876].

[49] J. Ambjorn, M. Flensburg, and C. Peterson, Langevin Simulations of Configurations With Static Charges, Phys. Lett. 159B (1985) 335-340.

[50] J. Ambjorn and S.-K. Yang, The SU(2) Chiral Model in an External Field: A Complex Stochastic Process on a Nonabelian Group, Nucl. Phys. B275 (1986) 18.

[51] J. Berges and I. O. Stamatescu, Simulating nonequilibrium quantum fields with stochastic quantization techniques, Phys. Rev. Lett. 95 (2005) 202003, [hep-1at/0508030].

[52] J. Berges, S. Borsanyi, D. Sexty, and I. O. Stamatescu, Lattice simulations of real-time quantum fields, Phys. Rev. D75 (2007) 045007, [hep-1at/0609058].

[53] J. Berges and D. Sexty, Real-time gauge theory simulations from stochastic quantization with optimized updating, Nucl. Phys. B799 (2008) 306-329, [arxiv:0708.0779]. 
[54] C. Pehlevan and G. Guralnik, Complex Langevin Equations and Schwinger-Dyson Equations, Nucl. Phys. B811 (2009) 519-536, [arxiv:0710.3756].

[55] G. Aarts and I.-O. Stamatescu, Stochastic quantization at finite chemical potential, JHEP 09 (2008) 018, [arXiv:0807.1597]].

[56] G. Aarts, Can stochastic quantization evade the sign problem? The relativistic Bose gas at finite chemical potential, Phys. Rev. Lett. 102 (2009) 131601, [arxiv:0810.2089].

[57] G. Aarts, Complex Langevin dynamics at finite chemical potential: Mean field analysis in the relativistic Bose gas, JHEP 05 (2009) 052, [arxiv:0902.4686].

[58] G. Aarts and K. Splittorff, Degenerate distributions in complex Langevin dynamics: one-dimensional QCD at finite chemical potential, JHEP 08 (2010) 017, [arXiv:1006.0332].

[59] G. Aarts and F. A. James, Complex Langevin dynamics in the SU(3) spin model at nonzero chemical potential revisited, JHEP 01 (2012) 118, [arXiv:1112.4655].

[60] D. Sexty, Simulating full QCD at nonzero density using the complex Langevin equation, Phys. Lett. B729 (2014) 108-111, [arxiv:1307.7748].

[61] T. Hayata and A. Yamamoto, Complex Langevin simulation of quantum vortices in a Bose-Einstein condensate, Phys. Rev. A92 (2015), no. 4 043628,

[62] Z. Fodor, S. D. Katz, D. Sexty, and C. Török, Complex Langevin dynamics for dynamical QCD at nonzero chemical potential: A comparison with multiparameter reweighting, Phys. Rev. D92 (2015), no. 9 094516, [arXiv: 1508.05260].

[63] G. Aarts, F. Attanasio, B. Jäger, and D. Sexty, The QCD phase diagram in the limit of heavy quarks using complex Langevin dynamics, JHEP 09 (2016) 087, [arxiv:1606.0556]].

[64] D. K. Sinclair and J. B. Kogut, Complex Langevin for Lattice QCD, PoS LATTICE2018 (2018) 143, [arXiv:1810.11880].

[65] F. Attanasio and B. Jäger, Dynamical stabilisation of complex Langevin simulations of QCD, Eur. Phys. J. C79 (2019), no. 1 16, [arXiv:1808.04400].

[66] K. Nagata, J. Nishimura, and S. Shimasaki, Complex Langevin calculations in finite density QCD at large $\mu / T$ with the deformation technique, Phys. Rev. D98 (2018), no. 11 114513, [arXiv:1805.03964].

[67] Y. Ito, H. Matsufuru, J. Nishimura, S. Shimasaki, A. Tsuchiya, and S. Tsutsui, Exploring the phase diagram of finite density QCD at low temperature by the complex Langevin method, PoS LATTICE2018 (2018) 146, [arxiv:1811.12688].

[68] J. Kogut and D. Sinclair, Applying complex langevin simulations to lattice qcd at finite density, Phys.Rev.D 100 (2019), no. 5 054512, [arXiv:1903.02622].

[69] D. Sexty, Calculating the equation of state of dense quark-gluon plasma using the complex langevin equation, Phys.Rev.D 100 (2019), no. 7 074503, [arxiv:1907.08712]].

[70] M. Hirasawa, A. Matsumoto, J. Nishimura, and A. Yosprakob, Complex Langevin analysis of 2D U(1) gauge theory on a torus with a $\theta$ term, arXiv:2004.13982.

[71] A. Mollgaard and K. Splittorff, Complex Langevin Dynamics for chiral Random Matrix Theory, Phys. Rev. D88 (2013), no. 11 116007, [arXiv:1309.4335]].

[72] A. Mollgaard and K. Splittorff, Full simulation of chiral random matrix theory at nonzero chemical potential by complex Langevin, Phys. Rev. D91 (2015), no. 3 036007, 
[73] J. Bloch, J. Glesaaen, J. J. M. Verbaarschot, and S. Zafeiropoulos, Complex Langevin Simulation of a Random Matrix Model at Nonzero Chemical Potential, JHEP 03 (2018) 015, [arXiv:1712.07514].

[74] P. Basu, K. Jaswin, and A. Joseph, Complex Langevin Dynamics in Large N Unitary Matrix Models, Phys. Rev. D98 (2018), no. 3 034501, [arxiv: 1802.1038]].

[75] A. Joseph and A. Kumar, Complex Langevin Simulations of Zero-dimensional Supersymmetric Quantum Field Theories, Phys. Rev. D100 (2019) 074507, [arXiv: 1908.04153].

[76] P. M. Stevenson, Optimized Perturbation Theory, Phys. Rev. D23 (1981) 2916.

[77] J. Nishimura, T. Okubo, and F. Sugino, Convergent Gaussian expansion method: Demonstration in reduced Yang-Mills integrals, JHEP 10 (2002) 043, [hep-th/0205253].

[78] H. Kawai, S. Kawamoto, T. Kuroki, and S. Shinohara, Improved perturbation theory and four-dimensional space-time in IIB matrix model, Prog. Theor. Phys. 109 (2003) 115-132, [hep-th/0211272].

[79] J. Nishimura, T. Okubo, and F. Sugino, Testing the Gaussian expansion method in exactly solvable matrix models, JHEP 10 (2003) 057, [hep-th/0309262].

[80] J. Nishimura, T. Okubo, and F. Sugino, Gaussian expansion analysis of a matrix model with the spontaneous breakdown of rotational symmetry, Prog. Theor. Phys. 114 (2005) 487-508, [hep-th/0412194].

[81] T. Aoyama, H. Kawai, and Y. Shibusa, Stability of 4-dimensional space-time from IIB matrix model via improved mean field approximation, Prog. Theor. Phys. 115 (2006) 1179-1187, [hep-th/0602244].

[82] T. Aoyama and Y. Shibusa, Improved perturbation method and its application to the IIB matrix model, Nucl. Phys. B754 (2006) 48-90, [hep-th/0604211].

[83] J. Nishimura and G. Vernizzi, Brane world from IIB matrices, Phys. Rev. Lett. 85 (2000) 4664-4667, [hep-th/0007022]. 